\title{
Mirror vision: Perceived size and perceived distance of virtual images
}

\author{
ATSUKI HIGASHIYAMA \\ Ritsumeikan University, Kyoto, Japan \\ and \\ KOICHI SHIMONO \\ Tokyo University of Marine Science and Technology, Tokyo, Japan
}

\begin{abstract}
We investigated spatial perception of virtual images that were produced by convex and plane mirrors. In Experiment 1, 36 subjects reproduced both the perceived size and the perceived distance of virtual images for five targets that had been placed at a real distance of 10 or $20 \mathrm{~m}$. In Experiment 2, 30 subjects verbally judged both the perceived size and the perceived distance of virtual images for five targets that were placed at each of five real distances of $2.5-45 \mathrm{~m}$. In both experiments, the subjects received objective-size and objective-distance instructions. The results were that (1) size constancy was attained for a distance of up to $45 \mathrm{~m}$, (2) distance was readily discriminated within this distance range, although virtual images produced by the mirror of strong curvature were judged to be farther away than those produced by the mirrors of less curvature, and (3) the ratio of perceived size to perceived distance was described as a power function of visual angle, and the ratio for the convex mirror was larger than that for the plane mirror. We compared the taking-into-account model and the direct perception model on the basis of a correlation analysis for proximal, virtual, and real levels of the stimuli. The taking-into-account model, which assumes that visual angle is transformed into perceived size by taking perceived distance into account, was supported by an analysis for the proximal level of stimuli. The direct perception model, which assumes that there is no inferential process between perceived size and perceived distance, was partially supported by an analysis for the distal level of the stimuli.
\end{abstract}

Mirrors generate virtual images of objects. In this study, we investigated the perceived size and the perceived distance of virtual images in convex and plane mirrors. The perception of virtual images in mirrors is a sort of transformed vision that is comparable to vision under water (e.g., Adolfson \& Berghage, 1974; Ono, O’Reiley, \& Herman, 1970; Ross, 1968; Ross \& Rejman, 1972; Vernoy, 1989; Vernoy \& Luria, 1977) or to a specific optical device (e.g., Harris, 1965; Held \& Gottlieb, 1958; Rock, 1966). However, interestingly enough, not much attention has been paid to mirror vision (Higashiyama, Yokoyama, \& Shimono, 2001).

Before mirror vision is considered, it will be useful to illustrate the nature of virtual images in mirrors. Figure 1 shows a convex mirror $\mathrm{CAB}$ with a radius of curvature, $2 f$. We call $f$ the focal distance and call $1 /(2 f)$ the curvature, $\kappa$. Consider the rays of light originating at an end-point $\mathrm{P}$ of an

This study was supported by research funds provided by Ritsumeikan University. We thank H. A. Sedgwick, who made very valuable comments on this article. Correspondence concerning this article should be addressed to A. Higashiyama, Department of Psychology, Ritsumeikan University, Kita-ku, Kyoto 603-8577, Japan (e-mail: achan@1t.ritsumei. ac.jp; for K.S., shimono@e.kaiyodai.ac.jp).

Note-This article was accepted by the previous editorial team, headed by Neil Macmillan. object PQ. The ray of light PN, which is parallel to the mirror axis $\mathrm{AO}$, is reflected at point $\mathrm{N}$ in the direction of point $\mathrm{T}$, so that points $\mathrm{T}$ and $\mathrm{N}$ are aligned with the focal point $\mathrm{F}$. The rays of light $\mathrm{PO}$ and $\mathrm{QO}$ return along the same course after being reflected by the mirror. It follows that line $N F$ intersects line $\mathrm{PO}$ at point $\mathrm{P}^{\prime}$. Line $\mathrm{P}^{\prime} \mathrm{Q}^{\prime}$, which is perpendicular to line $\mathrm{AO}$, is called the virtual image of the object PQ.

In this situation, it can be readily shown that

$$
z=\frac{y f}{f+y}
$$

and

$$
h=\frac{a x}{f}=\frac{a f}{f+y},
$$

where $x=\mathrm{Q}^{\prime} \mathrm{F}, y=\mathrm{QA}, z=\mathrm{AQ}^{\prime}, a=\mathrm{PQ}, h=\mathrm{P}^{\prime} \mathrm{Q}^{\prime}$, and $f=x+z$. We call $y$ the real distance of the object and $z$ its virtual distance; we call $a$ the real size of the object and $h$ its virtual size. Regardless of curvature, visual angle $\theta$ (in radians) of the virtual image is given by

$$
\theta=\arctan \frac{h}{z+d},
$$

where $d$ is the distance from the observer's eye to the mirror surface A (Figure 1). 


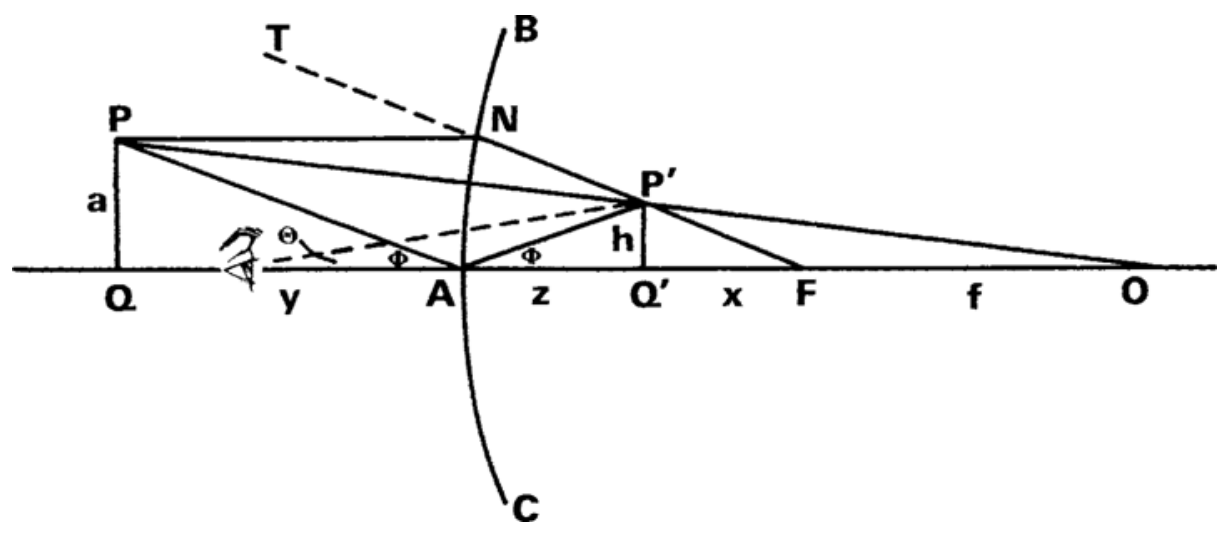

Figure 1. Optics of a convex mirror.

The left panel of Figure 2 illustrates the virtual distance as a function of real distance (i.e., Equation 1), and the right panel illustrates the virtual size of a $1-\mathrm{m}$ real object as a function of real distance (i.e., Equation 2). In each panel, $2 f$ was the parameter.

A plane mirror is a special case in which the focal distance is infinitely large. If $f$ is infinitely large in Equations 1 and 2, the virtual distance for the plane mirror is given by

$$
z=y,
$$

and the virtual size for the plane mirror is given by

$$
h=a \text {. }
$$

Equation 4 implies that the distance from the mirror surface to the virtual image is exactly the same as that from the mirror surface to the real objects, and Equation 5 implies that the virtual size in the plane mirror is exactly the same as the real size. Equations 4 and 5 are illustrated in Figure 2, together with the equations for convex mirrors.

Figure 2 shows that as curvature increases, the virtual size of an object is smaller than the real size (see the right panel), and the virtual distance is more packed in depth (see the left panel). This implies that the scenes in convex mirrors are miniature versions of real scenes in both size and depth. For intuitive comprehension, in Figure 3, we made sketches of virtual images in a plane mirror (left) and in a convex mirror with a $0.2-\mathrm{m}$ radius of curvature (right).

In this study, we first investigated how perceived size and perceived distance of single virtual images in a convex mirror differ from those in a plane mirror. If perceived size is determined by virtual size, we would predict that (1) virtual images in a convex mirror are perceived to be smaller than those in a plane mirror and (2) as a real object recedes from a convex or plane mirror, the virtual size
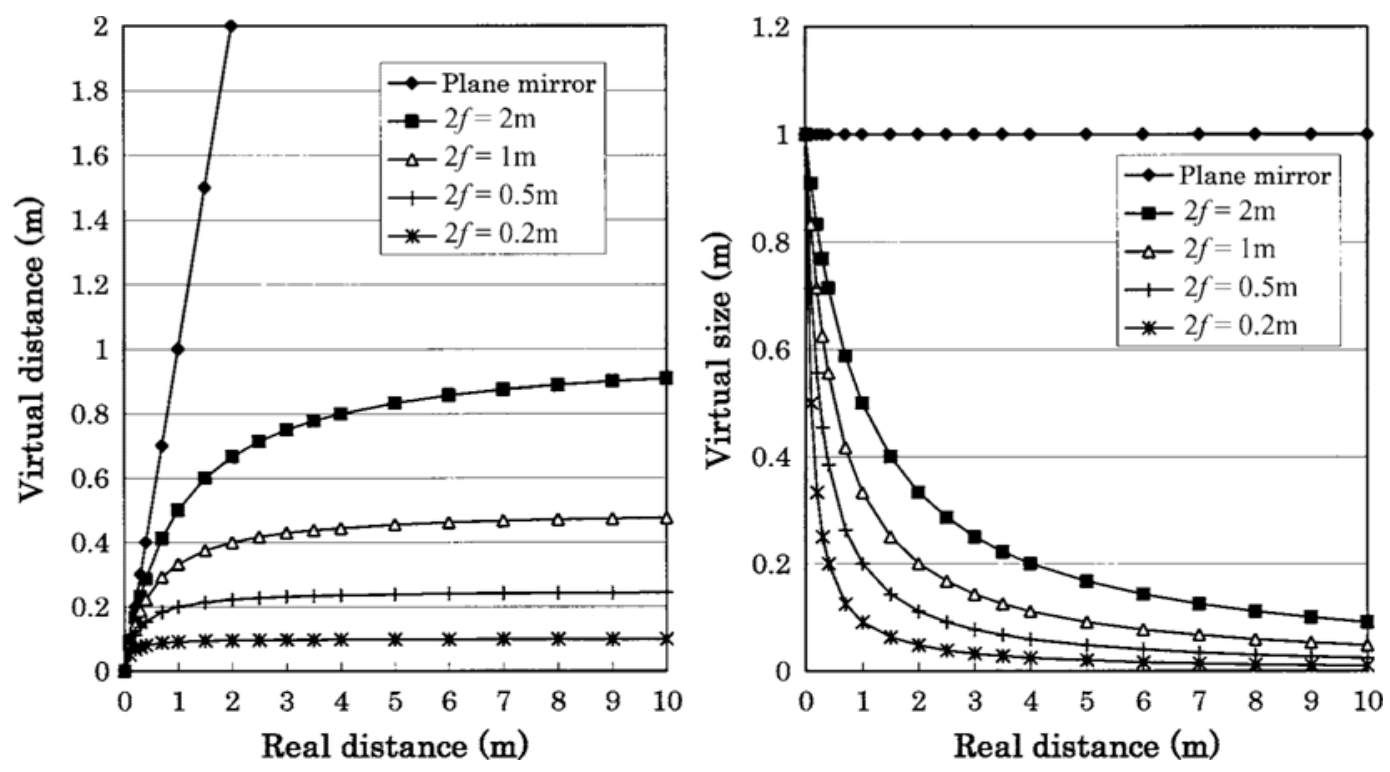

Figure 2. Virtual distance (left) and virtual size (right) of a 1-m target, as a function of real distance (in meters). The parameter is the radius of curvature $2 f$. 

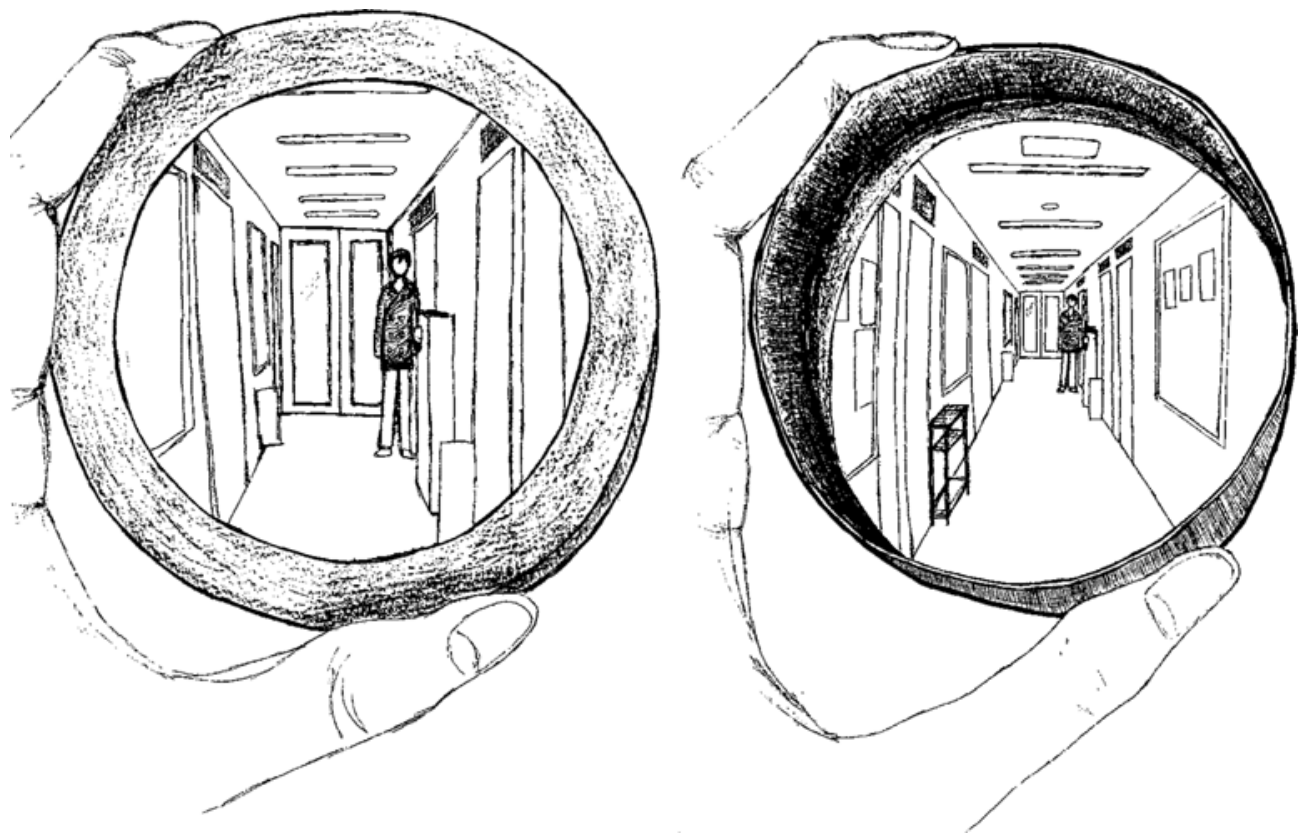

Figure 3. Sketches of a virtual scene in a plane mirror (left) and a convex mirror with $2 f=0.2 \mathrm{~m}$ (right).

in the convex mirror will be perceived to decrease rapidly, whereas virtual size in the plane mirror will be perceived to remain constant (see the right panel in Figure 2). Similarly, if perceived distance is determined by virtual distance, we would predict that (3) virtual distance in a convex mirror will be perceived to be smaller than that in a plane mirror and (4) as a real object recedes from a convex or plane mirror, the virtual distance in the convex mirror will be perceived to increase more slowly than that in the plane mirror (see the left panel in Figure 2).

Regardless of these optical predictions, the scenes in the mirrors do not appear as compressed as the optics of convex mirrors predicts. For example, when people drive cars while looking into convex mirrors, they do not seem to have much difficulty in judging the spatial layout of the automobiles on the road. Interestingly, we have actually found that virtual images in convex mirrors are localized over their optical positions and are judged to be farther away than those in a plane mirror (Higashiyama et al., 2001). These observations suggest that the perceived size and the perceived distance of virtual images do not follow the optics of mirrors.

Second, we investigated how well the size-distance invariance hypothesis (SDIH) describes the relation between perceived size and perceived distance in mirrors. The SDIH states that a retinal projection or visual angle of an object determines a unique ratio of apparent size to apparent distance (Epstein, Park, \& Casey, 1961; Sedgwick, 1986). The first attempt to formulate the SDIH mathematically (e.g., Kilpatrick \& Ittelson, 1953) was

$$
S^{\prime} / D^{\prime}=\tan \theta,
$$

where $S^{\prime}$ is the apparent size, $D^{\prime}$ is the apparent distance, and $\theta$ is the visual angle. For small $\theta$ s, Equation 6 approximates $S^{\prime} / D^{\prime}=\theta$.

Authors examining visual space perception (Baird \& Wagner, 1991; Gogel, 1998; Hershenson, 1992), haptic space perception (Barac-Cikoja \& Turvey, 1995), and space cognition (Hubbard, Kall, \& Baird, 1989) have developed their arguments on the basis of Equation 6. However, several studies (Gogel, Wist, \& Harker, 1963; Higashiyama \& Kitano, 1991; Vogel \& Teghtsoonian, 1972) have provided evidence that Equation 6 is too restrictive to describe the relations among $S^{\prime}, D^{\prime}$, and $\theta$. Alternatively, a number of studies (Foley, 1967, 1968; Higashiyama \& Shimono, 1994; Oyama, 1974) have assumed that $S^{\prime} / D^{\prime}$ is a power function of $\theta$ :

$$
S^{\prime} / D^{\prime}=K \theta^{n}
$$

where $K$ is the scale factor and $n$ is the exponent. In this study, we examined the validity of the SDIH by describing how $K$ and $n$ in Equation 7 vary with curvature.

Third, we investigated a perceptual process underlying mirror vision by means of partial correlation, which is a useful method by which to find a causal chain between stimulus variables and perceptual variables (Oyama, 1974, 1977; van der Meer, 1979). More specifically, we compared the taking-into-account model (Epstein, 1973; Gogel, 1973a, 1973b; Rock, 1975, 1984; Wallach \& Zuckerman, 1963) with the direct perception model (Gibson, 1950, 1979). The taking-into-account model assumes that we transform a visual angle into perceived size after taking perceived or registered distance into account. For the takinginto-account model, we assume a positive partial correla- 
tion between perceived size and perceived distance and also assume a positive partial correlation between visual angle and perceived size.

The direct perception model assumes that (1) perceived distance in natural everyday life is determined by the texture gradient of proximal stimuli and (2) perceived size of an object is determined, not by the simple visual angle of the object, but by a relation between the proximal stimuli that are formed by the object and the surrounds (e.g., the number of texture elements that are concealed by a target object [Gibson, 1950] or a relation of a target object to the horizontal line [Sedgwick, 1986]). Accordingly, for the direct perception model, we assume that there is a significant correlation between perceived size or distance and some higher order proximal stimulation, but we do not assume that there is a significant partial correlation between perceived size and perceived distance.

To compare these potent but incompatible models, we used partial correlation analysis, rather than multiple regression analysis. Multiple regression analysis reveals the relative effectiveness of stimulus variables that may contribute to a response variable. In particular, multiple regression analysis is useful at an early stage of research where we have to find truly effective stimuli from among many possible variables, but it does not reveal how these effective variables are connected in time sequence. In contrast, partial correlation analysis is useful at a later stage of research where it is needed to determine a suitable model from among several models that have competed to explain a perceptual phenomenon such as the size-distance relation. Both the taking-into-account model and the direct perception model have been well elaborated (Epstein, 1977; Sedgwick, 1986), and the largest difference between the two is whether an inferential or a cognitively significant link is assumed between perceived size and perceived distance.

In this study, we report two experiments in which subjects judged the perceived size and the perceived distance of virtual images. In Experiment 1, the subjects observed virtual images one at a time and reproduced the perceived size and the perceived distance of the virtual images. More specifically, the perceived distance of virtual images in mirrors was reproduced as the distance from the subject to a real person in a natural view, and the perceived size of the virtual images in mirrors was reproduced as the length of a tape in the subject's hands. In Experiment 2, in a more extended physical setting, the subjects verbally judged the perceived size and the perceived distance of the virtual images.

\section{EXPERIMENT 1}

\section{Method \\ Subjects. Thirty-six male university students volunteered as sub- jects.}

Stimuli and mirrors. The stimuli were five similar red rectangles $10 \times 15 \mathrm{~cm}, 20 \times 30 \mathrm{~cm}, 30 \times 45 \mathrm{~cm}, 40 \times 60 \mathrm{~cm}$, and $58 \times$ $72 \mathrm{~cm}$ (height by width). The four small rectangles were cut from foam polystyrene, and the largest rectangle was cut from paper, to reduce the weight of the stimulus. A magnet was fixed on the rear of each rectangle. With this magnet, each rectangle was fixed to a
$220 \mathrm{~cm}$ high $\times 3.5 \mathrm{~cm}$ diameter iron pole, which stood erect on a $39 \mathrm{~cm}$ wide $\times 10 \mathrm{~cm}$ deep $\times 10 \mathrm{~cm}$ high block at a distance of 10 or $20 \mathrm{~m}$ behind the subject.

We used two convex mirrors and one plane mirror. Each mirror had a diameter of $25 \mathrm{~cm}$. The radii of curvature for the convex mirrors were $0.65 \mathrm{~m}$ (strong curvature) and $1.0 \mathrm{~m}$ (weak curvature), and the radius of curvature for the plane mirror was infinitely large (zero curvature). These mirrors were placed, one at a time, on a frontal side of a $45.5 \mathrm{~cm}$ wide $\times 46.5 \mathrm{~cm}$ deep $\times 180 \mathrm{~cm}$ tall tower that was constructed from several aluminum pipes. The distance from the subject to the mirror was $50 \mathrm{~cm}$. The centers of the targets and the mirrors were $150 \mathrm{~cm}$ above the ground.

Equation 1 gives the distances of virtual images. The virtual distances for the real distance of $10 \mathrm{~m}$ were $0.31,0.48$, and $10 \mathrm{~m}$ for the mirrors with strong, weak, and zero curvature, respectively. The virtual distances for the real distance of $20 \mathrm{~m}$ were $0.32,0.49$, and $20 \mathrm{~m}$ for the mirrors of strong, weak, and zero curvature, respectively.

Equation 2 gives the sizes (widths) of virtual images. For the convex mirror with strong curvature, the virtual size of the targets varied from 5 to $23 \mathrm{~mm}$ for the real distance of $10 \mathrm{~m}$ and from 2 to $12 \mathrm{~mm}$ for the real distance of $20 \mathrm{~m}$. For the convex mirror with weak curvature, the virtual size of the targets varied from 7 to $34 \mathrm{~mm}$ for the real distance of $10 \mathrm{~m}$ and from 4 to $18 \mathrm{~mm}$ for the real distance of $20 \mathrm{~m}$. For the plane mirror, the virtual sizes of the targets were 15, 30, 45,60 , and $72 \mathrm{~cm}$ for both real distances.

Equation 3 gives the visual angles of virtual images under the assumption that $d=50 \mathrm{~cm}$. For the convex mirror with strong curvature, the visual angles of the virtual images varied from $0.17^{\circ}$ $(0.003 \mathrm{rad})$ to $1.59^{\circ}(0.028 \mathrm{rad})$; for the convex mirror with weak curvature, the visual angles of the virtual images varied from $0.21^{\circ}$ $(0.004 \mathrm{rad})$ to $2.01^{\circ}(0.035 \mathrm{rad})$; for the plane mirror, the visual angles of the virtual images varied from $0.42^{\circ}(0.007 \mathrm{rad})$ to $3.92^{\circ}$ (0.068 rad).

Procedure. The experiment was done in a $140 \times 140 \mathrm{~m}$ athletic field. Twelve subjects were randomly assigned to each of the three mirrors. When each subject arrived at the field, the experimenter required him to stand at the end of the field, directing his back to the field, and not to have any direct glimpse of the experimental setting in the field. The subject was permitted to see the field by having it reflected in the mirror, which was fixed on a stand at a distance of $50 \mathrm{~cm}$ in front of the subject. When the subject looked into the mirror, he saw not only a single target, but also several trees at the other end of the field, and he was also aware of buildings, the lawn on the ground, and a road behind the trees. In front of the subject, a male experimenter 166,170 , or $175 \mathrm{~cm}$ tall walked to or away from the subject. The subject saw the experimenter directly. The average eye level across subjects was $165 \mathrm{~cm}$ from the ground.

To measure the perceived size of the virtual images on a given trial, the subject adjusted the length of a tape ruler so that the length of the tape matched the perceived size (width) of the virtual images. The experimenter read the matched size and made a note of it. To measure the perceived distance of the virtual images, the subject gave verbal directions to the experimenter who approached or receded from the subject, so that the perceived distance from the subject to the experimenter matched the perceived distance from the subject to the virtual image. The experimenter read the matched distance with a tape ruler and made a note of it. The subject observed the standard or comparison target binocularly. Each subject was randomly coupled with one of the three experimenters. We did not treat experimenter as a factor in the analysis.

In the instructions to the subjects, the subjects were required to distinguish the real objects from their virtual images and to judge the objective size and the objective distance of the real objects that produced the virtual images. By objective size or objective distance, we mean the size or the distance that would be obtained if the subject measured it with a ruler, such as a meter stick (Gogel \& Da Silva, 1987; Higashiyama, Ishikawa, \& Tanaka, 1990; Kaess, 1980; Predebon, 1992). 

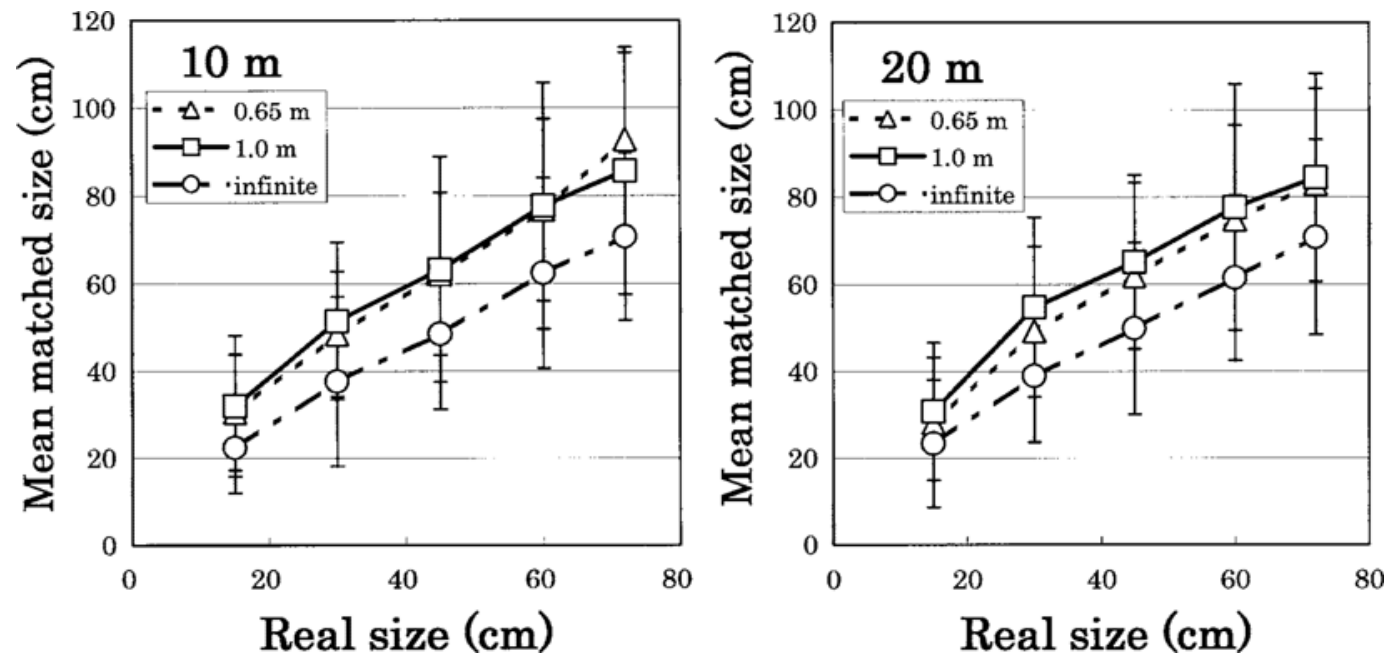

Figure 4. Mean matched size (in centimeters) as a function of real target size (in centimeters) for the 10-m standard distance (left panel) and for the 20-m standard distance (right panel). The parameter is the radius of curvature (in meters).

The trials consisted of 20 combinations of two distances, five targets, and two directions (approaching and receding). The order of the combinations was randomly determined for each subject. The subject judged both size and distance on each trial.

\section{Results and Discussion}

Figure 4 shows the mean size matches as a function of target size, with the mirror as the parameter. We performed a three-way (size $\times$ distance $\times$ mirror) analysis of variance (ANOVA) with repeated measures. The mean size matches increased as a function of real size $[F(4,132)=239.6, p<$ $.001]$, indicating that the subjects discriminated the target sizes in the mirror. The main effect of distance was not significant $[F(1,33)=2.0, p>.05]$. Although Figure 4 may give an impression that the mean size matches for the convex mirrors were larger than those for the plane mirror, this impression was not supported $[F(2,33)=2.5, p>.05]$. Thus, the perceived size of the virtual images was affected only by the real size.

Figure 5 shows the mean distance matches as a function of real distance, with the mirror as the parameter. We performed a three-way (size $\times$ distance $\times$ mirror) ANOVA with repeated measures. The mean distance matches for the 20-m distance were significantly different from those for the $10-\mathrm{m}$ distance $[F(1,33)=688.4, p<.001]$, indicating that the subjects distinguished the target distances in the mirror.

The main effect of mirror was significant $[F(2,33)=5.8$, $p<.01]$, indicating that the virtual images in the convex mirror with strong or weak curvature were perceived to be farther away than those in the plane mirror (Tukey's HSD test, $p<.05$ ).

The main effect of target size was significant $[F(4,132)=$ $6.4, p<.01]$. The mean distance matches for the targets $15,30,45,60$, and $72 \mathrm{~cm}$ wide were $12.7,12.4,12.3,11.8$, and $11.6 \mathrm{~m}$, respectively, for the $10-\mathrm{m}$ distance and 22.5 , $21.6,21.3,20.7$, and $20.5 \mathrm{~m}$, respectively, for the 20 -m distance. Clearly, other conditions being equal, small virtual images were perceived to be farther away than large virtual images.

Figure 6 shows $S^{\prime} / D^{\prime}$ as a function of $\theta$ in $\log$-log coordinates, with the real distance and the mirror as the parameters. Figure 6 suggests that for a given mirror, $S^{\prime} / D^{\prime}$ increases as a linear function of $\theta$ regardless of real target distance. We also note that $S^{\prime} / D^{\prime}$ was larger for the convex mirrors than for the plane mirror. For each mirror, we determined a least-square line that fitted the data points. We

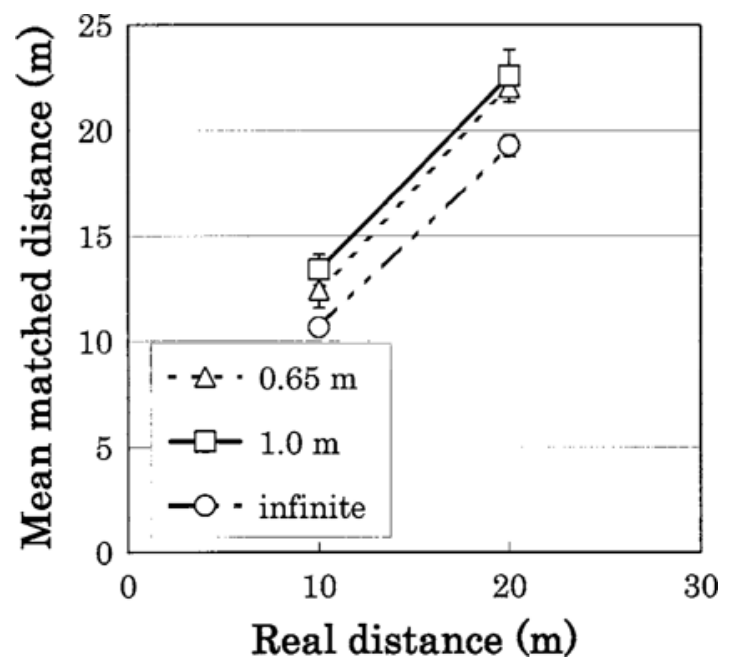

Figure 5. Mean matched distance (in meters) as a function of the real distance of the targets (in meters). The parameter is the radius of curvature (in meters). 
obtained $S^{\prime} / D^{\prime}=1.34 \theta^{0.80}$ for strong curvature, $S^{\prime} / D^{\prime}=$ $0.77 \theta^{0.72}$ for weak curvature, and $S^{\prime} / D^{\prime}=0.56 \theta^{0.79}$ for zero curvature. Thus, the exponent was almost the same across mirrors, but the scale factor increased with increasing curvature.

We performed three correlation analyses on the basis of proximal, virtual, and real levels of the stimuli. For the proximal level of the stimuli, the curvature $\kappa$ and the visual angle $\theta$ were used as stimulus variables for analysis, because these variables directly affect the geometric formation of the retinal image. For the virtual level of the stimuli, virtual size $S_{\mathrm{v}}$ and virtual distance $D_{\mathrm{v}}$ (exactly, virtual distance plus $50 \mathrm{~cm}$ ) of the virtual images were used as the stimulus variables for analysis. For the real level of the stimuli, objective size $S$ and objective distance $D$ of the real objects were used as the stimulus variables for analysis. For each analysis, the mean size matches and the mean distance matches were used as the response variables. Accordingly, two stimulus variables and two response variables were included in each of the analyses. All variables, except for curvature, were converted into logarithmic scores. In considering a partial correlation between two variables, we assumed the effects of the other variables to be kept constant.

Someone may argue that virtual size, virtual distance, real size, or real distance should not be included in causal analysis, because these variables are not the stimuli impinging directly on the eyes. Although this argument is very reasonable, nevertheless, we also used virtual or real variables for analysis, because these variables may be substitutes for some unknown higher order optical variables that determine perceived size and perceived distance.

Table 1 presents the results of correlation analyses. For the proximal level of the stimuli, we obtained a significant simple correlation between $\theta$ and $S^{\prime}$ and a significant sim- ple correlation between $\theta$ and $D^{\prime}$, and we also obtained a significant partial correlation for every pair of variables examined. The results of the partial correlations suggest that (1) $\theta$ affects both $S^{\prime}$ and $D^{\prime},(2) \kappa$ affects both $S^{\prime}$ and $D^{\prime}$, and (3) $S^{\prime}$ affects $D^{\prime}$ and vice versa. These findings support the taking-into-account model, which assumes that there is a positive partial correlation between $\theta$ and $S^{\prime}$ and a positive partial correlation between $S^{\prime}$ and $D^{\prime}$. However, these findings pose a challenge for the direct perception model, which does not assume a significant partial correlation between $S^{\prime}$ and $D^{\prime}$.

A more direct test of the direct perception model is obtained from the results for the virtual or the real level of stimuli, because the stimulus variables involved in this analysis are assumed to be proxies for unknown higher order optical variables. It was found that the partial correlation between $S^{\prime}$ and $D^{\prime}$ was significantly medium (.61) for the virtual level of the stimuli and was significantly high (.90) for the real level of the stimuli. It is difficult to interpret these results with the direct perception model.

In addition to illustrating an examination of perceptual process models, Table 1 indicates several findings worthy of note. Both simple and partial correlations between $\theta$ and $D^{\prime}$ were significantly negative for the proximal level of the stimuli. This effect of visual angle on perceived distance has been known as the relative-size cue (Epstein \& Landauer, 1969; Gogel, 1964, 1969; Landauer \& Epstein, 1969). Although the relative-size cue to perceived distance has been considered effective in reduced conditions of viewing (Higashiyama, 1977), the results of this experiment suggest that it is effective in a natural view seen in mirrors.

The effect of curvature on perceived size was opposite to that of visual angle. The virtual images in the convex mirror formed small visual angles, and therefore, they were perceived to be smaller than the virtual images in the plane

Table 1

Simple and Partial Correlations Among Curvature $\kappa$, Visual Angle $\theta$, Perceived Size $\boldsymbol{S}^{\prime}$, and Perceived Distance $D^{\prime}$

\begin{tabular}{ccc}
\hline Paired Var.; Controlled Var. & Simple Correlation & Partial Correlation \\
\hline$\left(\kappa, S^{\prime} ; \theta, D^{\prime}\right)$ & Proximal Level & \\
$\left(\theta, S^{\prime} ; \kappa, D^{\prime}\right)$ & .26 & $.98^{* *}$ \\
$\left(\kappa, D^{\prime} ; \theta, S^{\prime}\right)$ & $.57^{* *}$ & $.99^{* *}$ \\
$\left(\theta, D^{\prime} ; \kappa, S^{\prime}\right)$ & .23 & $-.95^{* *}$ \\
$\left(S^{\prime}, D^{\prime} ; \theta, \kappa\right)$ & $-.62^{* *}$ & $-.97^{* *}$ \\
& -.02 & $.96^{* *}$ \\
$\left(S_{\mathrm{v}}, S^{\prime} ; D_{\mathrm{v}}, D^{\prime}\right)$ & Virtual Level & \\
$\left(D_{\mathrm{v}}, S^{\prime}, S_{\mathrm{v}}, D^{\prime}\right)$ & .03 & $.73^{* *}$ \\
$\left(S_{\mathrm{v}}, D^{\prime} ; D_{\mathrm{v}}, S^{\prime}\right)$ & -.26 & $-.76^{* *}$ \\
$\left(D_{\mathrm{v}}, D^{\prime} ; S_{\mathrm{v}}, S^{\prime}\right)$ & $-.39^{*}$ & $-.84^{* *}$ \\
$\left(S^{\prime}, D^{\prime} ; S_{\mathrm{v}}, D_{\mathrm{v}}\right)$ & -.02 & $.81^{* *}$ \\
& -.02 & $.61^{* *}$ \\
$\left(S, S^{\prime} ; D, D^{\prime}\right)$ & Real Level & \\
$\left(D, S^{\prime} ; S, D^{\prime}\right)$ & $.95^{* *}$ & $.99^{* *}$ \\
$\left(S, D^{\prime} ; D, S^{\prime}\right)$ & -.01 & $-.89^{* *}$ \\
$\left(D, D^{\prime} ; S, S^{\prime}\right)$ & -.10 & $-.91^{* *}$ \\
$\left(S^{\prime}, D^{\prime} ; S, D\right)$ & $.95^{* *}$ & $.99^{* *}$ \\
\hline
\end{tabular}

Note $-N=30$. Var. $=$ variables. $\quad * p<.05 . \quad * * p<.01$. 


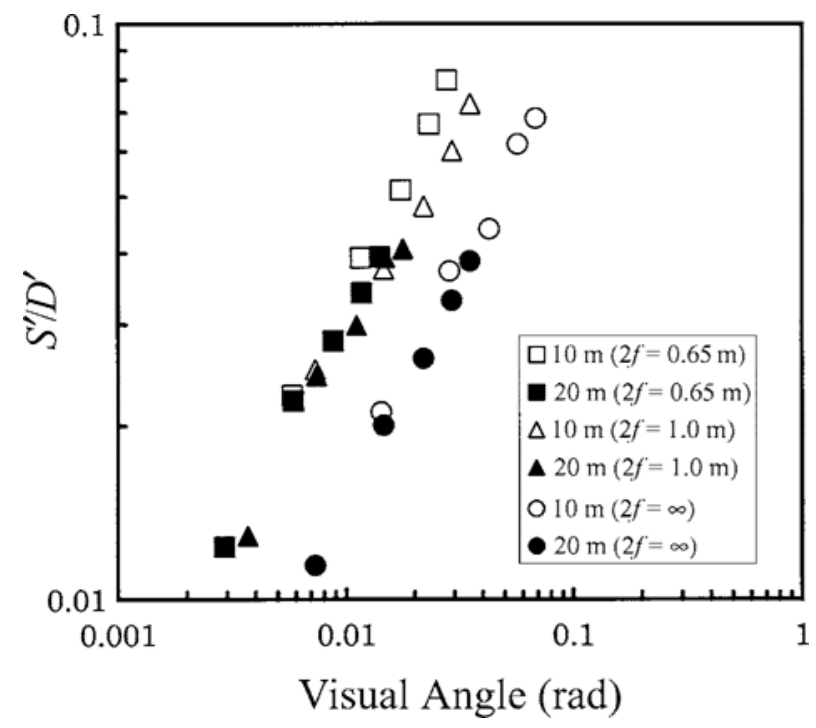

Figure 6. $S^{\prime} / D^{\prime}$ as a function of visual angle (in radians). The parameter is the radius of curvature (in meters) and the real distance of targets (in meters).

mirror, as the positive partial correlation between $\theta$ and $S^{\prime}$ suggests. However, the virtual images in the convex mirror were judged to be larger than those in the plane mirror, as the positive partial correlation between $\kappa$ and $S^{\prime}$ suggests. This correlation between $\kappa$ and $S^{\prime}$ can be interpreted as a compensation for the small visual angles that were produced by the convex mirrors.

The effect of curvature on perceived distance was also opposite to that of visual angle. We obtained a significant negative partial correlation between $\theta$ and $D^{\prime}$ and a significant negative partial correlation between $\kappa$ and $D^{\prime}$. The former suggests that because of the small visual angles, the virtual images in the convex mirrors were perceived to be farther than those in the plane mirror, whereas the latter suggests that the virtual images in the convex mirror were judged to be closer than those in the plane mirror. This correlation between $\kappa$ and $D^{\prime}$ can also be interpreted as a compensation for the enlargement of perceived distance that was produced by the small visual angle in the convex mirrors. The effects of compensation will be discussed later on.

\section{EXPERIMENT 2}

In Experiment 2, we attempted to confirm the results of Experiment 1, in a more extended physical setting, with the method of absolute estimation (i.e., verbal estimation using physical units). In Experiment 1, we found that (1) there is a strong tendency of size constancy for both convex and plane mirrors, (2) $S^{\prime} / D^{\prime}$ increases linearly with visual angle on log-log coordinates, but for a given visual angle, $S^{\prime} / D^{\prime}$ is larger for the convex mirrors than for the plane mirror, and (3) the taking-into-account model gives a better fit to data than does the direct perception model.
In Experiment 2, we also attempted to establish a scale for distance in mirrors. Since we compared five target distances in this experiment, we were able to specify how perceived distance grows as a function of real distance (note that we had used two distances in Experiment 1). According to the principles for the optical position of a virtual image, a virtual image in a convex mirror has to be perceived to be closer than that in a plane mirror, and as a real object recedes from a convex or plane mirror, the perceived distance of the virtual image in the convex mirror will increase more slowly than that in the plane mirror. We examined whether these optical principles regarding distance work well for mirrors.

With several partial correlation analyses, we again sought a network of information processing among stimulus variables and response variables. Our concern was to see how well the pattern of partial correlations in Experiment 1 would be reproduced in Experiment 2, which differed in stimulus setting and measurement method.

\section{Method}

Subjects. Thirty university students ( 15 males and 15 females) volunteered as subjects. They were newly recruited, and none of them had participated in Experiment 1.

Stimuli and mirrors. The targets were five similar red triangles $32 \times 16 \mathrm{~cm}, 48 \times 24 \mathrm{~cm}, 72 \times 36 \mathrm{~cm}, 108 \times 54 \mathrm{~cm}$, and $162 \times$ $81 \mathrm{~cm}$ (height by base). Each target was cut from plywood and stood directly on the ground, with a support on the rear of the target. Each target was presented at a distance of $2.5,5,15,30$, or $45 \mathrm{~m}$ behind the subject. Therefore, there were 25 combinations of target and distance.

We used two convex mirrors and one plane mirror. Each mirror had a diameter of $20 \mathrm{~cm}$. The radii of curvature for the convex mirrors were $0.22 \mathrm{~m}$ (strong curvature) and $0.60 \mathrm{~m}$ (weak curvature), and the radius of curvature for the plane mirror was infinitely large (zero curvature).

For the convex mirror with strong curvature, the virtual distances of the targets varied from 10.5 to $11.0 \mathrm{~cm}$; for the convex mirror with weak curvature, the virtual distances varied from 26.8 to $29.8 \mathrm{~cm}$; for the plane mirror, the virtual distances varied from 2.5 to $45 \mathrm{~m}$.

For the convex mirror with strong curvature, the virtual sizes of the targets varied from 1 to $68 \mathrm{~mm}$; for the convex mirror with weak curvature, the virtual sizes varied from 0.2 to $17.4 \mathrm{~cm}$; for the plane mirror, the virtual sizes varied from $0.32 \mathrm{~m}$ to $1.62 \mathrm{~m}$.

For the convex mirror with strong curvature, the visual angles of the virtual images varied from $0.11^{\circ}(0.002 \mathrm{rad})$ to $9.63^{\circ}(0.168 \mathrm{rad})$; for the convex mirror with weak curvature, the visual angles varied from $0.20^{\circ}(0.004 \mathrm{rad})$ to $17.38^{\circ}(0.303 \mathrm{rad})$; for the plane mirror, the visual angles varied from $0.40^{\circ}(0.007 \mathrm{rad})$ to $32.29^{\circ}(0.563 \mathrm{rad})$. These calculations of visual angles were done under the assumption that $d=30 \mathrm{~cm}$.

Procedure. Ten subjects were randomly assigned to each of the three mirrors. The experiment was done outdoors in a $1.7 \mathrm{~m}$ wide $\times$ $50 \mathrm{~m}$ long corridor. One side of the corridor was a uniform concrete wall, and the other side was a series of windows on a building wall. When the subject arrived at the corridor, the experimenter required him or her to stand at one end of the corridor, directing his or her back to it, and not to have any glimpse of the experimental setting. Each subject grasped the mirror with the preferred hand or both hands and reflected a single target in the mirror. The subject was permitted to move the head freely and also to move the hands that were used to grasp the mirror. Yet the subject was prevented from seeing the targets directly.

The subject saw the virtual images one at a time. The subject verbally judged both the perceived size (height) and the perceived dis- 
tance of the virtual image. The subject judged size with the unit of centimeters and judged distance with the unit of meters. The subject was instructed to judge the objective size and the objective distance of the real targets that produced the virtual images (i.e., objective instructions). After the subject had finished the judgments on a given trial, the experimenter changed the target for the next trial. While the targets were being changed, the subject did not see the mirror.

The order of 25 target-distance combinations was randomly determined for each subject. Half the subjects judged size after judging distance for each target, and the remaining subjects judged them in the reverse order.

\section{Results and Discussion}

Since the distribution of verbal judgments was positively skewed, we obtained the geometric means of judgments. Figure 7 shows the results for size estimations. The left, middle, and right panels represent the results for strong, weak, and zero curvatures, respectively. In each panel, the mean size estimation is plotted against real distance, with the target size as the parameter. We performed a three-way (size $\times$ distance $\times$ mirror) ANOVA with repeated measures. The main effect of size was significant $[F(4,108)=$ $640.9, p<.001]$. The main effect of distance was not significant; the main effect of mirror was not significant. Figure 7 clearly shows that size constancy was well achieved for each mirror.

Figure 8 shows the geometric means of the distance estimations. The left, middle, and right panels represent the results for strong, weak, and zero curvatures, respectively. In each panel, the mean distance estimation is plotted against the real distance, with the real size as the parameter. We performed a three-way (size $\times$ distance $\times$ mirror)
ANOVA with repeated measures. The main effect of distance was significant $[F(4,108)=466.6, p<.001]$, but the main effect of size was not significant. Thus, for any mirror used, the judged distance increased with increasing real distance, regardless of the real size.

The main effect of mirror was significant $[F(2,27)=3.44$, $p<.05]$. The interaction between mirror and distance was not significant. Figure 8 indicates that the virtual images in the mirror with strong curvature were judged to be farther than those in the mirrors with less curvature. To scrutinize the effect of mirror, we assumed a power function $D^{\prime}=a D^{m}$ between real distance $D$ and perceived distance $D^{\prime}$ and estimated $a$ and $m$ with a least-square criterion. We obtained $D^{\prime}=1.13 D^{0.90}$ for strong curvature, $D^{\prime}=1.01 D^{0.87}$ for weak curvature, and $D^{\prime}=0.55 D^{1.03}$ for zero curvature. Clearly, the exponent for the convex mirrors was smaller than that for the plane mirror, and the scale factor increased with increasing curvature. These equations are shown in Figure 9, where the geometric mean of distance estimation is plotted against real distance on log-log coordinates.

Figure 10 shows $S^{\prime} / D^{\prime}$ as a function of $\theta$, with the mirror as the parameter. Clearly, the $S^{\prime} / D^{\prime}$ for the convex mirrors is generally larger than that for the plane mirror. The leastsquare lines that fitted the data points were $S^{\prime} / D^{\prime}=$ $2.15 \theta^{0.92}$ for strong curvature, $S^{\prime} / D^{\prime}=2.07 \theta^{0.95}$ for weak curvature, and $S^{\prime} / D^{\prime}=2.12 \theta^{1.14}$ for zero curvature. We note that the exponent decreased with increasing curvature.

In the same manner as that in Experiment 1, we performed correlation analyses for the three levels of stimuli. Table 2 presents the results. For the proximal level of the
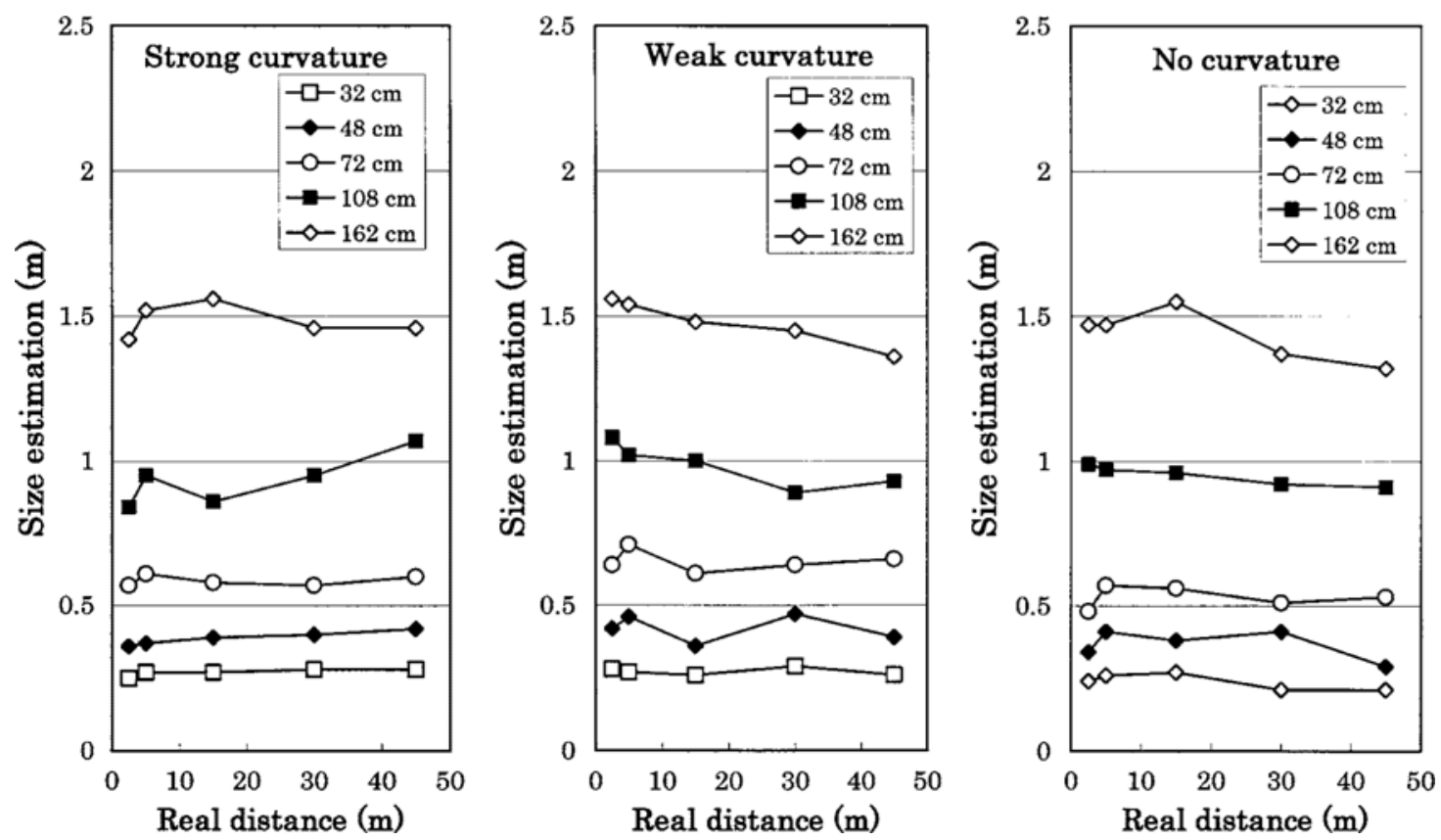

Figure 7. Mean size estimation (in meters) as a function of the real distance of the targets (in meters). The left, center, and right panels represent the strong $(2 f=0.22 \mathrm{~m})$, weak $(2 f=0.60 \mathrm{~m})$, and zero curvatures of a mirror. In each panel, the parameter is the real size of the targets (in centimeters). 

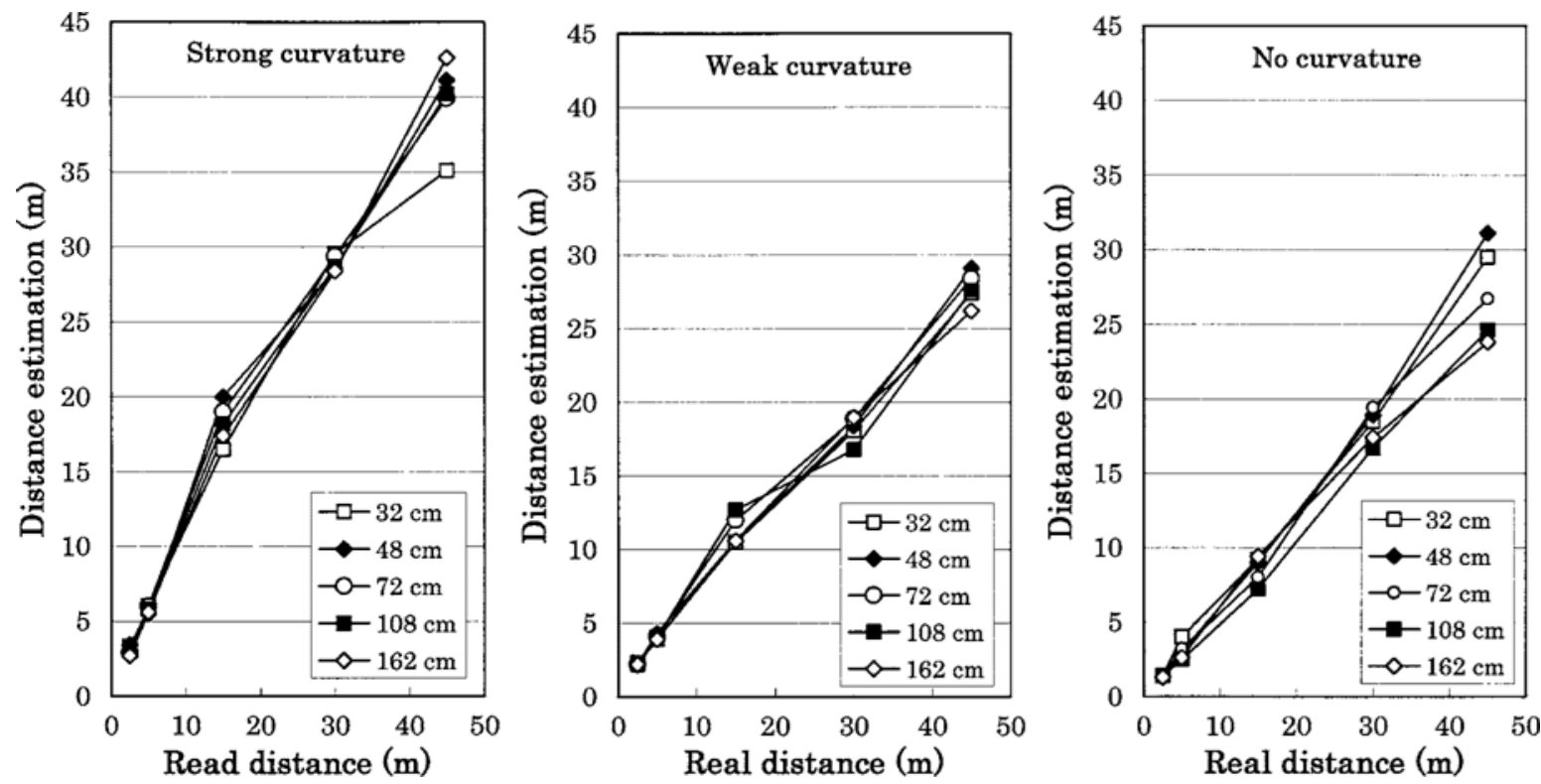

Figure 8. Mean distance estimation (in meters) as a function of the real distance of the targets (in meters). The left, center, and right panels represent the strong $(2 f=0.22 \mathrm{~m})$, weak $(2 f=0.60 \mathrm{~m})$, and zero curvatures of a mirror. In each panel, the parameter is the real size of the targets (in centimeters).

stimuli, we obtained a significant simple correlation between $\theta$ and $S^{\prime}$ and a significant simple correlation between $\theta$ and $D^{\prime}$ and we also obtained a significant partial correlation for almost every pair of variables examined. The results of partial correlations suggest that (1) $\theta$ affects both $S^{\prime}$ and $D^{\prime},(2) \kappa$ affects $S^{\prime}$ but does not affect $D^{\prime}$, and (3) $S^{\prime}$ affects $D^{\prime}$ and vice versa. These results are in contrast with the results of Experiment 1, where visual angle and curvature equally affected perceived size and perceived distance. It seems that the effects of visual angle on perceived size and perceived distance were equal to or larger than the effects of curvature on them.

For the virtual level of the stimuli, we obtained a significant simple correlation between $S_{\mathrm{v}}$ and $D^{\prime}$ and obtained a significant partial correlation between $S^{\prime}$ and $D^{\prime}$. It is difficult to interpret these findings with the direct perception model. These results are the same as the results in Experiment 1 .

For the real level of stimuli, we obtained significant simple and partial correlations between $S$ and $S^{\prime}$ and obtained significant simple and partial correlations between $D$ and $D^{\prime}$. Yet there was not a significant simple or partial correlation between $S^{\prime}$ and $D^{\prime}$. These results agree with the direct perception model, differing from the results in Experiment 1 .

\section{GENERAL DISCUSSION}

\section{Mediational or Direct?}

The results of correlation analysis for the proximal level of the stimuli were mostly the same as those in Experi- ments 1 and 2 . The outcomes in common were that (1) $\theta$ was positively correlated with $S^{\prime}$ and negatively correlated with $D^{\prime}$, and $\kappa$ was positively correlated with $S^{\prime}$ and (2) there was a positive correlation between $S^{\prime}$ and $D^{\prime}$. These results fit the taking-into-account model but pose a challenge to the direct perception model.

The correlation analysis for the virtual or the real level of the stimuli is useful for examining the direct perception model. For the virtual level of the stimuli, the partial cor-

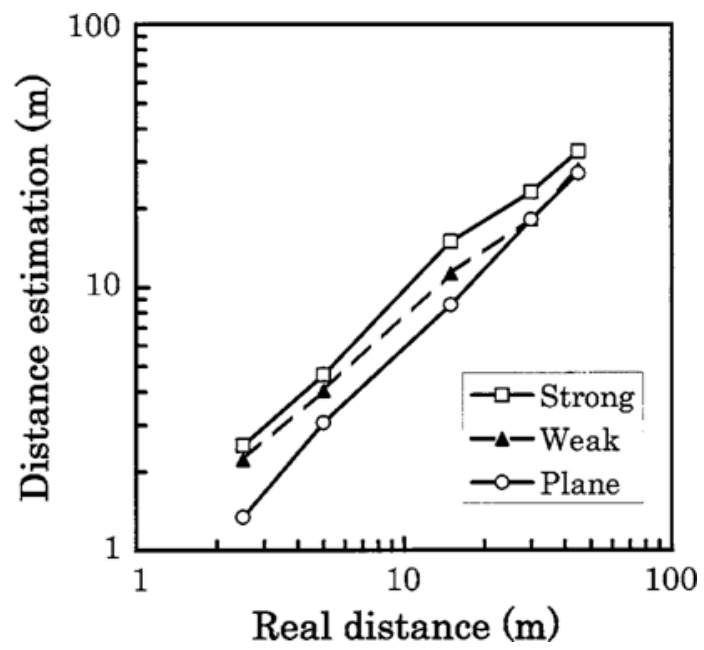

Figure 9. Mean distance estimation (in meters) as a function of the real distance of the targets (in meters) on log-log coordinates. The parameter is the curvature. 


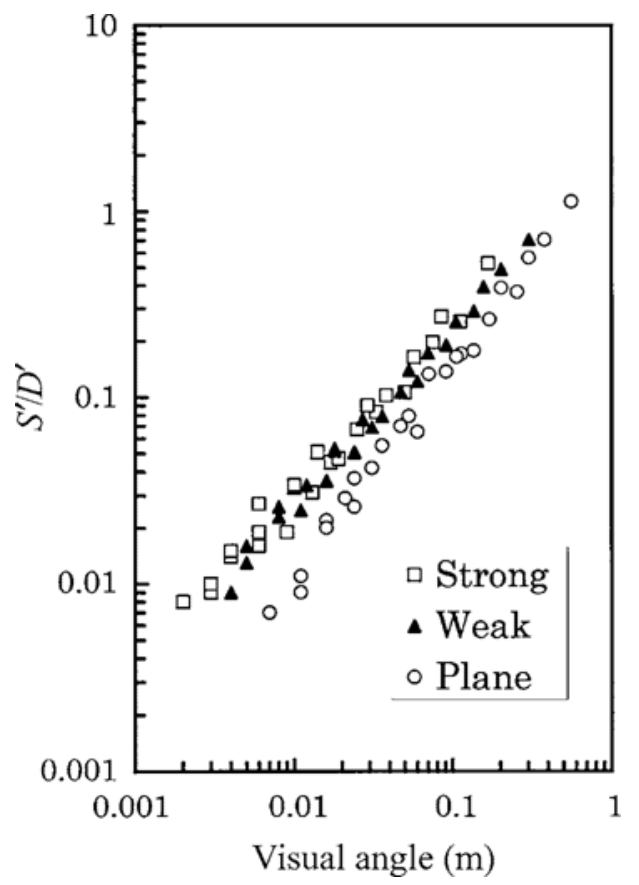

Figure 10. $S^{\prime} / D^{\prime}$ as a function of visual angle (in radians). The parameter is the curvature.

relation between $S^{\prime}$ and $D^{\prime}$ was significant in both experiments, implying that the direct perception model was not suitable. For the real level of the stimuli, the partial correlation between $S^{\prime}$ and $D^{\prime}$ was significant in Experiment 1 but was not significant in Experiment 2. This result implies that the direct perception model was valid in Experiment 2.
To summarize, we obtained significant partial correlations between $S^{\prime}$ and $D^{\prime}$ in five of six correlation analyses (i.e., 2 experiments $\times 3$ levels). This means that the takinginto-account model, or a general mediational process model, was generally supported in this study.

One may ask why the mediational process prevailed in this study. We think that instructions to the subjects are a critical factor that activates the mediational process. Several studies with reaction time paradigms (Broota \& Epstein, 1973; Epstein \& Broota, 1975) have suggested that objective instructions, such as those used in this study, activate an inferential process. In particular, Epstein and Broota found that when subjects judged the size of targets under objective or phenomenal size instructions, the reaction time needed for estimating objective size increased with the increasing viewing distance of the targets, whereas the reaction time needed for phenomenal size estimation was smaller than that for objective size estimation and was the same for all viewing distances. From these findings, the authors concluded that a cognitive operation is involved in objective size estimation.

One may also ask why the direct perception model was supported only for the real level of the stimuli in Experiment 2. One possible explanation has been suggested by Norman (1980), who studied the reaction times necessary for size judgments of two targets that were presented at different viewing distances. The author found that for targets that differed largely in distal size, the reaction time was almost constant regardless of the exocentric distance (i.e., depth) between the targets, whereas for the targets that were similar in distal size, the reaction time decreased as the exocentric distance increased. These findings suggest that direct perception of size occurred for the targets that differed largely in distal size. It should be noted that

Table 2

Simple and Partial Correlations Among Curvature $\kappa$, Visual Angle $\theta$, Perceived Size $S^{\prime}$, and Perceived Distance $D^{\prime}$

\begin{tabular}{ccc}
\hline Tested Var.; Controlled Var. & Simple Correlation & Partial Correlation \\
\hline$\left(\kappa, S^{\prime} ; \theta, D^{\prime}\right)$ & Proximal Level & \\
$\left(\theta, S^{\prime} ; \kappa, D^{\prime}\right)$ & .04 & $.47^{*}$ \\
$\left(\kappa, D^{\prime} ; \theta, S^{\prime}\right)$ & $.46^{*}$ & $.95^{* *}$ \\
$\left(\theta, D^{\prime} ; \kappa, S^{\prime}\right)$ & .24 & -.44 \\
$\left(S^{\prime}, D^{\prime} ; \theta, \kappa\right)$ & $-.89^{* *}$ & $-.99^{* *}$ \\
& -.03 & $.94^{* *}$ \\
$\left(S_{\mathrm{v}}, S^{\prime} ; D_{\mathrm{v}}, D^{\prime}\right)$ & Virtual Level & \\
$\left(D_{\mathrm{v}}, S^{\prime} ; S_{\mathrm{v}}, D^{\prime}\right)$ & .20 & $.97^{* *}$ \\
$\left(S_{\mathrm{v}}, D^{\prime} ; D_{\mathrm{v}}, S^{\prime}\right)$ & .07 & $-.97^{* *}$ \\
$\left(D_{\mathrm{v}}, D^{\prime} ; S_{\mathrm{v}}, S^{\prime}\right)$ & $-.48^{*}$ & $-.99^{* *}$ \\
$\left(S^{\prime}, D^{\prime} ; S_{\mathrm{v}}, D_{v}\right)$ & .04 & $.99^{* *}$ \\
& -.03 & $.98^{* *}$ \\
$\left(S, S^{\prime} ; D, D^{\prime}\right)$ & Real Level & \\
$\left(D, S^{\prime} ; S, D^{\prime}\right)$ & $.99^{* *}$ & $.99^{* *}$ \\
$\left(S, D^{\prime} ; D, S^{\prime}\right)$ & -.02 & -.19 \\
$\left(D, D^{\prime} ; S, S^{\prime}\right)$ & -.02 & -.18 \\
$\left(S^{\prime}, D^{\prime} ; S, D\right)$ & $.96^{* *}$ & $.96^{* *}$ \\
\hline
\end{tabular}

Note $-N=75$. Var. $=$ variables. $\quad * p<.05 . \quad * * p<.01$. 
in the present study, both the size and the distance ranges for the targets in Experiment 2 were definitely larger than those in Experiment 1.

We do not believe that a single process (i.e., the mediational or the direct process) is exclusively involved in activation in all situations of space perception. As was suggested above, two factors seem to regulate the activation of the size-distance processes. One factor is the discrimination of size or distance: The more difficult the discrimination, the more the mediational process is involved (Norman, 1980). In mirror vision, it is difficult to discriminate the size and the distance of virtual images, because of the shrunken size and the compressed distance of the virtual images. Another factor is the attitude of viewing: When the objective attitude of viewing is emphasized in the perceiving of size and distance, the mediational process is enhanced. It seems that a task that requires accurate estimation of size and distance evokes a complicated inferential process.

\section{Size}

Virtual images in plane mirrors provide an accurate duplication of the real world, whereas virtual images in convex mirrors provide a distorted miniature version of the real world. Despite this difference in the nature of the mirrors, the judgments of perceived size and perceived distance were not or were little affected by curvature. The judgments of perceived size were kept constant up to the real distance of $45 \mathrm{~m}$ for both the plane and the convex mirrors. These results for the plane mirror are not amazing, because in realistic environments, size constancy is well achieved (Higashiyama et al., 1990; Teghtsoonian, 1970, 1974). However, these results for the convex mirrors may be surprising if we realize that as a real object recedes from the convex mirror, the virtual image in it diminishes (e.g., when $2 f=0.22 \mathrm{~m}$, a target $162 \mathrm{~cm}$ tall produces virtual images of 13 and $8 \mathrm{~mm}$ for the real distances of 2.5 and $45 \mathrm{~m}$, respectively). These results pose a challenge for the first and second optical predictions mentioned in the introduction.

The direct perception approach and the mediational process approach assume different explanations of size constancy. The direct perception approach has often assumed that if the relation in angular size between a target and its surrounds is the same independently of viewing distance, size constancy is maintained (Rock \& Ebenholtz, 1959; Sedgwick, 1986). This is illustrated in Figure 3, in which the angular relation of a target (e.g., a person) to its surrounds (e.g., a door) is almost the same for plane mirrors and convex mirrors. This explanation of size constancy is simply applicable to both normal vision and mirror vision, but it does not fully explain the network of processing that is suggested by the partial correlations in Tables 1 and 2.

Alternatively, the results of partial correlation analyses suggest that two mediational processes work in mirror vision. One is the process in which visual angle is converted into perceived size by taking perceived distance into ac- count. This is proved by the fact that the partial correlation between $\theta$ and $S^{\prime}$ and the partial correlation between $D^{\prime}$ and $S^{\prime}$ were significant. Another is the process in which visual angle is converted into perceived size by taking curvature into account. As is indicated in Tables 1 and 2, the effect of curvature on perceived size was opposite to that of visual angle. Since perceived size is positively correlated with visual angle, the targets in a convex mirror are perceived to be smaller than those in a plane mirror. But at the same time, the visual system takes curvature into account and compensates for the small visual angles of virtual images in a convex mirror (i.e., the positive correlation between curvature and perceived size). As a net result of these conflicting effects of curvature and visual angle, size constancy may be maintained in mirrors. Although both the direct perception approach and the mediational process approach are theoretically possible, the results of partial correlation analysis suggest that the mediational process model gives a better fit to the data than the direct perception approach does.

\section{Distance}

The distance judgments were very nearly veridical with respect to the real environment. Although virtual images in convex mirrors are optically localized within the focal distance, the judged distances in both experiments were definitely greater than the focal distance and approached the real distance of the targets. This finding is clearly at variance with the optical localization.

More interesting, virtual images in convex mirrors were perceived to be farther away than those in the plane mirror. This result poses a challenge to the third prediction mentioned in the introduction: Virtual distance in a convex mirror is perceived to be smaller than that in a plane mirror. To account for this result, we assume that the subjects did not rely on oculomotor adjustments that may be induced by virtual images but probably made use of pictorial cues. Among the pictorial cues, the visual angles of a virtual image and its surrounds seem to be effective for perceived distance, even if the viewing distance to the virtual images is $1 \mathrm{~m}$ or less.

The growth of perceived distance in convex mirrors was more compressed than that in plane mirrors. In Experiment 2, the exponents of the power function for the convex mirrors $(0.90$ and 0.87$)$ were smaller than the exponent for the plane mirrors (1.03). This result is in agreement with the fourth prediction mentioned in the introduction: As a real object recedes from a convex or a plane mirror, virtual distance in the convex mirror is perceived to increase more slowly than that in the plane mirror.

How can we explain the fact that the exponent for convex mirrors was less than unity, whereas the exponent for plane mirrors was about unity? One possible explanation is based on the optical property of mirrors that virtual distance in convex mirrors increases with a negative acceleration, whereas virtual distance in plane mirrors increases linearly (see the left panel of Figure 2). In particular, the convex mirrors used in this study optically made the scene 
so packed that it was almost a two-dimensional scene like a picture plane (i.e., the exocentric distance between the farthest and the closest virtual images was several centimeters). This explanation reminds us of Hayashibe's (2002) study, in which subjects judged the perceived egocentric distance of objects in an actual scene and in two artificial scenes (i.e., a video-recorded stereoscopic scene and a computer-controlled virtual reality scene). In these settings, the artificial scenes contained fewer cues to distance than did the actual scene, or the artificial scenes contained many more cues indicating flatness of scene. When a power function was fitted to the distance judgments, the exponents of the power function were $0.96-1.01$ for the actual scene, $0.53-0.75$ for the video-recorded stereoscopic scene, and $0.57-0.80$ for the virtual reality scene. Both the present and Hayashibe's studies demonstrated that the fewer the cues to distance, the more compressed the perceived distance (i.e., small exponents).

A different explanation of distance compression is possible from the meditational process approach: Distance judgments are cognitive for convex mirrors, whereas they are perceptual for plane mirrors. For a convex mirror, subjects would have to infer distance by correcting the distortion of virtual images, but for a plane mirror, such cognitive correction is not needed. On the basis of a review of studies of psychological distance, Wiest and Bell (1985) showed that when distance was judged with direct viewing, memory, and inference, the exponents of the power functions applied to the distance judgments were 1.09 , 0.91 , and, 0.75 , respectively. We do not know which of the optical and the cognitive explanations is better.

\section{SDIH}

The results of both experiments indicated that $S^{\prime} / D^{\prime}$ increased linearly with visual angle on log-log coordinates, but for a given visual angle, $S^{\prime} / D^{\prime}$ for the convex mirror was generally larger than that for the plane mirror. This finding suggests that $S^{\prime} / D^{\prime}$ is determined not only by visual angle, but also by other factors. This result reminds us of previous studies in which the invariance of $S^{\prime} / D^{\prime}$ has been doubted. Gogel et al. (1963) showed that for a given $\theta, S^{\prime} / D^{\prime}$ is a curvilinear function of target distance, and $S^{\prime} / D^{\prime}$ is also increased by a modification of binocular disparity that can be accomplished by magnification of the effective interpupillary distance of the eyes. Vogel and Teghtsoonian (1972) showed that although $S^{\prime} / D^{\prime}$ is a monotonically increasing function of visual angle, it also depends on the structure of the texture gradient. Higashiyama and Kitano (1991) demonstrated that other conditions being equal, $S^{\prime} / D^{\prime}$ is larger for neutral targets than for familiar persons. Higashiyama and Shimono (1994) argued that $S^{\prime} / D^{\prime}$ is larger in a reduced condition of viewing than in a normal condition of viewing. All of these studies have indicated that size and distance judgments are not influenced uniformly by environmental and task variables.

Since the values $n$ and $K$ in the SDIH vary in an extremely complicated way, it is difficult to summarize them succinctly.
However, the average $n$ value in Experiments 1 and 2 was smaller for convex mirrors $(n=0.85)$ than for plane mirrors $(n=0.97)$, whereas the average $K$ value was larger for the convex mirrors $(K=1.58)$ than for the plane mirrors $(K=1.34)$. Thus, curvature affects both the exponent and the scale factor of the power function. We also note that both $n$ and $K$ were generally smaller in Experiment 1 than in Experiment 2: In Experiment 1, the power function, averaged over mirrors, was $S^{\prime} / D^{\prime}=0.89 \theta^{0.77}$, whereas in Experiment 2, it was $2.11 \theta^{1.01}$. These results suggest that $n$ and $K$ also depend on such factors as the physical setting of the targets and the method of measurement.

Finally, we emphasize that perception of size and distance in mirrors is very nearly veridical with respect to the environment: The visual world in a convex mirror is almost the same as that in a plane mirror. It is also interesting that the adaptive visual world is attained the moment one looks into a mirror. One does not need much time to construct the normalized visual world from the distorted optical structure. We do not think that a sort of perceptual adaptation, such as prism adaptation, was in progress during the experiments, because on each trial, after being exposed to a distorted scene in a convex mirror, the subjects saw the normal scene while the targets were changed for the next trial. Therefore, throughout the experiments, the subjects were aware of the difference between the normal and the distorted visual worlds. We think that there is a mechanism that makes the distorted optical structure correspond to the normal visual world, without a long-lasting learning of the relation between a proximal stimulation and the visual world.

\section{REFERENCES}

Adolfson, J., \& Berghage, T. (1974). Perception and performance under water. New York: Wiley.

BAIRD, J. C., \& WAGneR, M. (1991). Transformation theory of size judgment. Journal of Experimental Psychology: Human Perception \& Performance, 17, 852-864.

Barac-CikoJa, D., \& Turvey, M. T. (1995). Does perceived size depend on perceived distance? An argument from extended haptic perception. Perception \& Psychophysics, 57, 216-224.

Broota, K. D., \& Epstein, W. (1973). The time it takes to make vertical size and distance judgments. Perception \& Psychophysics, 14, 358-364.

EPSTEIN, W. (1973). The process of "taking-into-account" in visual perception. Perception, 2, 267-285.

EPSTEIN, W. (1977). Historical introduction to constancies. In W. Epstein (Ed.), Stability and constancy in visual perception: Mechanisms and processes (pp. 1-22). New York: Wiley.

Epstein, W., \& Broota, K. D. (1975). Attitude of judgment and reaction time in estimation of size at a distance. Perception \& Psychophysics, 18, 201-204.

Epstein, W., \& Landauer, A. A. (1969). Size and distance judgments under reduced conditions of viewing. Perception \& Psychophysics, 6 , 269-272.

Epstein, W., Park, J., \& Casey, A. (1961). The current status of the size-distance hypotheses. Psychological Bulletin, 58, 491-514.

FOLEY, J. M. (1967). Binocular disparity and perceived relative distance: An examination of two hypotheses. Vision Research, 7, 655-670.

Foley, J. M. (1968). Depth, size and distance in stereoscopic vision. Perception \& Psychophysics, 3, 265-274. 
GiBson, J. J. (1950). The perception of the visual world. Boston: Houghton Mifflin.

Gibson, J. J. (1979). The ecological approach to visual perception. Boston: Houghton Mifflin.

Gogel, W. C. (1964). Size cue to visually perceived distance. Psychological Bulletin, 62, 217-235.

GoGEL, W. C. (1969). The sensing of retinal size. Vision Research, 9 , 1079-1094.

GoGEL, W. C. (1973a). The organization of perceived space: I. Consequences of perceptual interactions. Psychologische Forschung, $\mathbf{3 6}_{2}$ 195-221.

GoGEL, W. C. (1973b). The organization of perceived space: II. Perceptual interactions. Psychologische Forschung, 36, 223-247.

Gogel, W. C. (1998). An analysis of perceptions from changes in optical size. Perception \& Psychophysics, 60, 805-820.

Gogel, W. C., \& DA SiLVA, J. A. (1987). Familiar size and the theory of off-sized perceptions. Perception \& Psychophysics, 41, 318-328.

Gogel, W. C., Wist, E. R., \& Harker, G. S. (1963). A test of the invariance of the ratio of perceived size to perceived distance. American Journal of Psychology, 76, 537-553.

HARRIS, C. S. (1965). Perceptual adaptation to inverted, reversed, and displaced vision. Psychological Review, 72, 419-444.

HayAshibe, K. (2002). Apparent distance in actual, three-dimensional video-recorded, and virtual reality. Perceptual \& Motor Skills, 95 , 573-582.

HELD, R., \& GotTlieb, N. (1958). A technique for studying adaptation to disarranged hand-eye coordination. Perceptual \& Motor Skills, 8, 83-86.

HERSHENSON, M. (1992). Size-distance invariance: Kinetic invariance is different from static invariance. Perception \& Psychophysics, 51, 541-548.

Higashiyama, A. (1977). Perceived size and distance as a perceptual conflict between two processing modes. Perception \& Psychophysics, 22, 206-211.

Higashiyama, A., IshiKawa, T., \& TAnaka, K. (1990). Visual alleys as a function of instructions under informative and reduced conditions of viewing. Perception \& Psychophysics, 47, 468-476.

Higashiyama, A., \& Kitano, S. (1991). Perceived size and distance of persons in natural outdoor settings: The effects of familiar size. Psychologia, 34, 188-199.

HigashiYama, A., \& Shimono, K. (1994). How accurate is size and distance perception for very far terrestrial objects? Function and causality. Perception \& Psychophysics, 55, 429-442.

Higashiyama, A., Yokoyama, Y., \& Shimono, K. (2001). Perceived distance of targets in convex mirrors. Japanese Psychological Research, 43, 13-24.

HuBbard, T. L., Kall, D., \& Baird, J. C. (1989). Imagery, memory, and size-distance invariance. Memory \& Cognition, 17, 87-94.

KaEss, D. W. (1980). Instructions and decision times of size-constancy responses. Perception \& Psychophysics, 27, 477-482.

Kilpatrick, F. P., \& ITtelson, W. H. (1953). The size-distance invariance hypothesis. Psychological Review, 60, 223-231.

LANDAUER, A. A., \& EPSTEIN, W. (1969). Does retinal size have a unique correlate in perceived size? Perception \& Psychophysics, 6, 273-275.
Norman, J. (1980). Direct and indirect perception of size. Perception \& Psychophysics, 28, 306-314.

Ono, H., O'Reiley, J., \& Herman, L. (1970). Underwater distance distortion within a manual work space. Human Factors, 12, 473-480.

Oyama, T. (1974). Perceived size and perceived distance in stereoscopic vision and an analysis of their causal relations. Perception \& Psychophysics, 16, 175-181.

OYAMA, T. (1977). Analysis of causal relations in the perceptual constancies. In W. Epstein (Ed.), Stability and constancy in visual perception (pp. 183-216). New York: Wiley.

Predebon, J. (1992). The role of instructions and familiar size in absolute judgments of size and distance. Perception \& Psychophysics, 51, 344-354.

Rock, I. (1966). The nature of perceptual adaptation. New York: Basic Books.

Rock, I. (1975). An introduction to perception. New York: Macmillan.

Rock, I. (1984). Perception. New York: Scientific American Books.

Rock, I., \& Ebenholtz, S. (1959). The relational determination of perceived size. Psychological Review, 66, 387-401.

Ross, H. E. (1968). Water, fog and the size-distance invariance hypothesis. British Journal of Psychology, 58, 301-313.

Ross, H. E., \& REJMAN, M. H. (1972). Adaptation to speed distortions under water. British Journal of Psychology, 63, 257-264.

SEDGwick, H. A. (1986). Space perception. In K. R. Boff, L. Kaufman, \& J. P. Thomas (Eds.), Handbook of perception and human performance (Vol. 1, pp. 21-1 to 21-57). New York: Wiley.

Teghtsoonian, M. (1970). The effects of size and distance on magnitude estimations of apparent size. American Journal of Psychology, 83, 601-612.

TEGHTSOONIAN, M. (1974). The doubtful phenomenon of over-constancy. In H. R. Moskowitz, B. Scharf, \& J. C. Stevens (Eds.), Sensation and measurement: Papers in honor of S. S. Stevens (pp. 411-420). Dordorecht: Reidel.

VAN DER MeER, H. C. (1979). Interrelation of the effects of binocular disparity and perspective cues on judgments of depth and height. Perception \& Psychophysics, 26, 481-488.

Vernoy, M. W. (1989). Simultaneous adaptation to size, distance, and curvature underwater. Human Factors, 31, 77-85.

Vernoy, M. W., \& LuRIA, S. M. (1977). Perception of, and adaptation to, a three-dimensional curvature distortion. Perception \& Psychophysics, 22, 245-248.

Vogel J. M., \& Teghtsoonian, M. (1972). The effects of perspective alterations on apparent size and distance scales. Perception \& Psychophysics, 11, 294-298.

Wallach, H., \& Zuckerman, C. (1963). The constancy of stereoscopic depth. American Journal of Psychology, 76, 404-412.

WIEST, W. M., \& BELL, B. (1985). Stevens's exponent for psychophysical scaling of perceived, remembered, and inferred distance. Psychological Bulletin, 98, 457-470.

(Manuscript received December 30, 2002; revision accepted for publication September 12, 2003.) 